\title{
Effect of eukaryotic translation initiation factor 4A3 in malignant tumors (Review)
}

\author{
YUANHANG ZHU, CHENCHEN REN and LI YANG \\ Department of Obstetrics and Gynecology, The Third Affiliated Hospital of \\ Zhengzhou University, Zhengzhou, Henan 450052, P.R. China \\ Received August 11, 2020; Accepted February 19, 2021
}

DOI: $10.3892 / \mathrm{ol} .2021 .12619$

\begin{abstract}
Eukaryotic translation initiation factor 4A3 (EIF4A3), a key component of the exon junction complex, is widely involved in RNA splicing and nonsense-mediated mRNA decay. EIF4A3 has also been reported to be involved in cell cycle regulation and apoptosis. Thus, EIF4A3 may serve as a pivotal regulatory factor involved in the occurrence and development of multiple diseases. Previous studies have demonstrated that EIF4A3 is mutated in neuromuscular degenerative lesions and is differentially expressed in several tumors, serving as a non-coding RNA binding protein to regulate its expression. In addition, studies have reported that inhibiting EIF4A3 can prevent tumor cell proliferation, thus, several researchers are trying to design and synthesize potent and selective EIF4A3 inhibitors. The present review summarizes the function of EIF4A3 in cell cycle and discusses it underlying molecular mechanisms that contribute to the occurrence of malignant diseases. In addition, EIF4A3 selective inhibitors, and bioinformatics analyses performed to analyze the expression and mutations of EIF4A3 in gynecological tumors and breast cancer, are also discussed.
\end{abstract}

\section{Contents}

1. Introduction

2. EIF4A3 and cell function

3. EIF4A3 and tumors

4. EIF4A3 inhibitors

Correspondence to: Dr Chenchen Ren, Department of Obstetrics and Gynecology, The Third Affiliated Hospital of Zhengzhou University, 7 Front Kangfu Street, Zhengzhou, Henan 450052, P.R. China

E-mail: renchenchen1106@126.com

Abbreviations: EIF4A3, eukaryotic translation initiation factor 4A3; DEAD, Asp-Glu-Ala-Asp; EJC, exon junction complex; NMD, nonsense-mediated mRNA decay; HCC, hepatocellular carcinoma; GBM, glioblastoma multiforme; GC, gastric cancer; HTS, high-throughput screening

Key words: EIF4A3, EJC, NMD, inhibitors
5. Bioinformatics analysis of EIF4A3 in gynecological tumors and breast cancer

6. Conclusions

\section{Introduction}

Eukaryotic translation initiation factor 4A3 (EIF4A3), a member of the EIF4A Asp-Glu-Ala-Asp (DEAD)-box and ATP-dependent RNA helicase families (1-4), serves as a translation initiation factor by unraveling secondary structures of the 5'-untranslated region (5). In 2004, EIF4A3 was reported as a novel and vital element of the exon junction complex (EJC), which binds spliced but not intronless mRNAs by anchoring the EJC to RNA (6). The EJC is a complex that contains multiple proteins and serves different functions, including splicing and polyadenylation (7), mRNA export $(8,9)$, nonsense-mediated mRNA decay (NMD) (10), regulation of translation efficiency (11) and the localization of mRNA (12). EIF4A3 is situated $\sim 24$ nucleotides upstream of the exon junction, which is the canonical EJC region, but can also be found in noncanonical regions within the exons (13). NMD is an important process that ensures accurate and efficient translation of proteins, and prevents the synthesis of abnormal or defective proteins by degrading incomplete or defective mRNA (14). The key event in inducing NMD is phosphorylation of the trans-acting factor, UPF1 RNA helicase and ATPase (UPF1), by SMG1 NMD associated PI3K related kinase (15). The translation termination release factors, eukaryotic translation termination factor (ERF)1 and ERF3, and the EJC are required for UPF1 phosphorylation and the occurrence of NMD (15). RNA interference experiments have demonstrated that nonsense codon-induced partitioning shift depends on certain NMD factors, including UPF1 and EIF4A3, but not the UPF3B regulator of NMD (16). EIF4A3 is an essential component in the physiological processes of humans. The present review discusses current literature on the function of EIF4A, particularly in tumors.

\section{EIF4A3 and cell function}

A previous study reported that the spliceosomal factor, CWC22 spliceosome associated protein homolog (CWC22), directly interacts with EIF4A3 to recruit the EJC to targeted mRNA (17). Ryu et al (18) revealed the detailed mechanism, 
demonstrating that threonine 163 in the RNA-binding motif of EIF4A3 can be phosphorylated by CDK1 and CDK2 in a cell cycle-dependent manner, which prevents binding of EIF4A3 to spliced mRNAs and other EJC members, and promotes the interaction between EIF4A3 and CWC22. However, this phosphorylation inhibits NMD. The splicing of mRNA relies on the RNA binding motif protein 8A (Y14)/mago homolog, EJC subunit (Magoh) heterodimer, the core component of EJC, which is located on the corresponding target site. The process also requires the participation of EIF4A3. However, the location of other components of the EJC is not affected by EIF4A3, which suggests that the binding of different components of the EJC to mRNA involves a different signaling pathway. Furthermore, EIF4A3 has been reported to be associated with spliced mRNAs at the EJC, suggesting that EIF4A3 may provide an association between splicing and translation in the cytoplasm (6). Furthermore, computational screening has demonstrated that EIF4A3 is a potential regulator for mRNA-protein expression level discrepancy (19). Thus, EIF4A3 is an indispensable molecule in protein translation.

Previous studies have reported that EIF4A3 is a key component of cell cycle and apoptosis regulation (20-23). In addition, EJC-related components serve a vital role in the splicing of apoptosis factor mRNA. For example, recombinant EIF4A3, Y14 and Magoh proteins preferentially bind to the endogenous apoptotic factor BCL2 like 1 (Bcl-x) precursor RNA, and specifically transfer Bcl-x alternative splicing to the nuclear extract (20). Furthermore, EIF4A3 serves a role in cell cycle monitoring. Inhibition of EIF4A3 using compounds or gene interference technology decreases cell cycle arrest in the $\mathrm{G}_{2} / \mathrm{M}$ phase, which in turn increases apoptosis (21).

\section{EIF4A3 and tumors}

Bioinformatics analyses have revealed that EIF4A3 expression is upregulated at the transcriptional level in common malignant tumors (24). E59K/Q is the most prevalent mutation in the DEAD domain, which influences the TNF- $\alpha / \mathrm{NF}-\kappa \mathrm{B}$ signaling pathway (24). Patients with tumors frequently produce autoantibodies, and the identification of tumor autoantigens may serve a role in the early diagnosis and immunotherapy of cancer (25). EIF4A3 reactivity has been observed in patients with pancreatic, colorectal, gastric and hepatocellular cancers, but has not been observed in patients with chronic pancreatitis or lung cancer, or in healthy individuals, which suggests that EIF4A3 may serve as a serum diagnostic marker for patients with cancer (26). In hepatocellular carcinoma (HCC), phosphorylation of EIF4A3 is associated with metastasis by regulating mRNA splicing, and other spliceosome proteins have also been reported to be involved in the process (27). Lin et al (28) demonstrated that EIF4A3 expression is upregulated in HCC tissues compared with healthy liver tissues, and high EIF4A3 expression is associated with a poor prognosis. EIF4A3 is strongly associated with the expression of several types of cell cycle regulatory genes (CDK1 and CDK2), tumor-associated transcription factors, chemokine signaling pathways and spliceosome signaling pathways $(18,20-22)$. In addition, EIF4A3 expression is upregulated in ovarian cancer tissues compared with adjacent healthy ovarian tissues (23). However, in patients with pregnancy-associated breast cancer, EIF4A3 expression is downregulated, as determined via database analysis (29).
Taken together, these findings suggest that the pathogenic mechanisms of EIF4A3 vary in different types of cancer.

EIF4A3, as an RNA binding protein (RBP), regulates the expression of non-coding RNAs in tumors. In glioblastoma multiforme (GBM), circ-matrix metallopeptidase (MMP)9 serves as an oncogene and is associated with cell proliferation, migration and invasion, among which EIF4A3 promotes circMMP9 expression by interacting with the upstream region of the circMMP9 mRNA transcript (30). In breast cancer, EIF4A3 modulates the cell cycle by promoting the expression of circ-septin 9 (SEPT9) by binding to SEPT9 pre-mRNA (22). In gastric cancer (GC), hsa_circ_001988 attenuates GC progression, and EIF4A3 serves as an RNA-binding protein to promote hsa_circ_001988 expression (31). Another study reported that VCAN antisense RNA 1 interacts with EIF4A3 to prevent EIF4A3 from recruiting tumor protein p53 mRNA, which downregulates p53 expression in GC cells (32). In breast cancer and GC, bioinformatics analyses have demonstrated that EIF4A3 is an RBP to circular RNA $(33,34)$. In addition, EIF4A3 has been identified as a long non-coding RNA cancer susceptibility 2 (CASC2)-binding protein, which inhibits SKOV3 ovarian cancer cell viability, migration and invasion, and increases cell apoptosis by regulating CASC2 (23). Another study in GBM demonstrated that EIF4A3 extends the half-life of long intergenic non-protein coding RNA (LINC)00680 and TTN antisense RNA 1 (TTN-AS1) (35). Similar to LINC00680 and TTN-AS1 knockdown, EIF4A3 knockdown inhibits glioblastoma cell proliferation, migration and invasion, and increases apoptosis (35). In non-small cell lung cancer, LINC00667 is recruited to EIF4A3 to stabilize vascular endothelial growth factor A mRNA (36). Furthermore, in pancreatic adenocarcinoma, LINC01232 is recruited to EIF4A3 to regulate the mRNA stability of transmembrane 9 superfamily member 2 (TM9SF2), which regulates TM9SF2 protein expression (37).

\section{EIF4A3 inhibitors}

In 2011, a pan EIF4A inhibitor, hippuristanol, was reported to inhibit human $\mathrm{T}$ lymphotrophic virus type 1-infected T-cell line and adult T-cell leukemia cell proliferation, but not normal peripheral blood mononuclear cell proliferation, by inducing cell cycle arrest at the $G_{1}$ phase and decreasing the expression of cell cycle protein and cyclin-dependent kinase, and promoting apoptosis by decreasing the expression levels of Bcl-x, baculoviral IAP repeat containing 3, X-linked inhibitor of apoptosis and CASP8 and FADD like apoptosis regulator (38). Recent studies have demonstrated that NMD inhibition induces tumor immunity and enhances cancer chemotherapy $(39,40)$. Based on the key role of EIF4A3 in NMD and the therapeutic potential of targeting EIF4A3 in cancer, several research groups are pursuing the development of EIF4A3 inhibitors.

There are three natural products that can be combined with EIF4A, including hippuristanol, pateamine A and rocaglates (41). Hippuristanol displays decreased effectiveness toward EIF4A3, requiring 10 -fold higher concentrations compared with EIF4A1/2 (42). Nuclear magnetic resonance analysis revealed that hippuristanol inhibits EIF4A1 by binding to its allosteric site (42). Pateamine A is a pan inhibitor for EIF4A, which blocks EIF4F complex (used for translation initiation) formation by stabilizing the interaction between EIF4A and targeted RNA (43). However, 

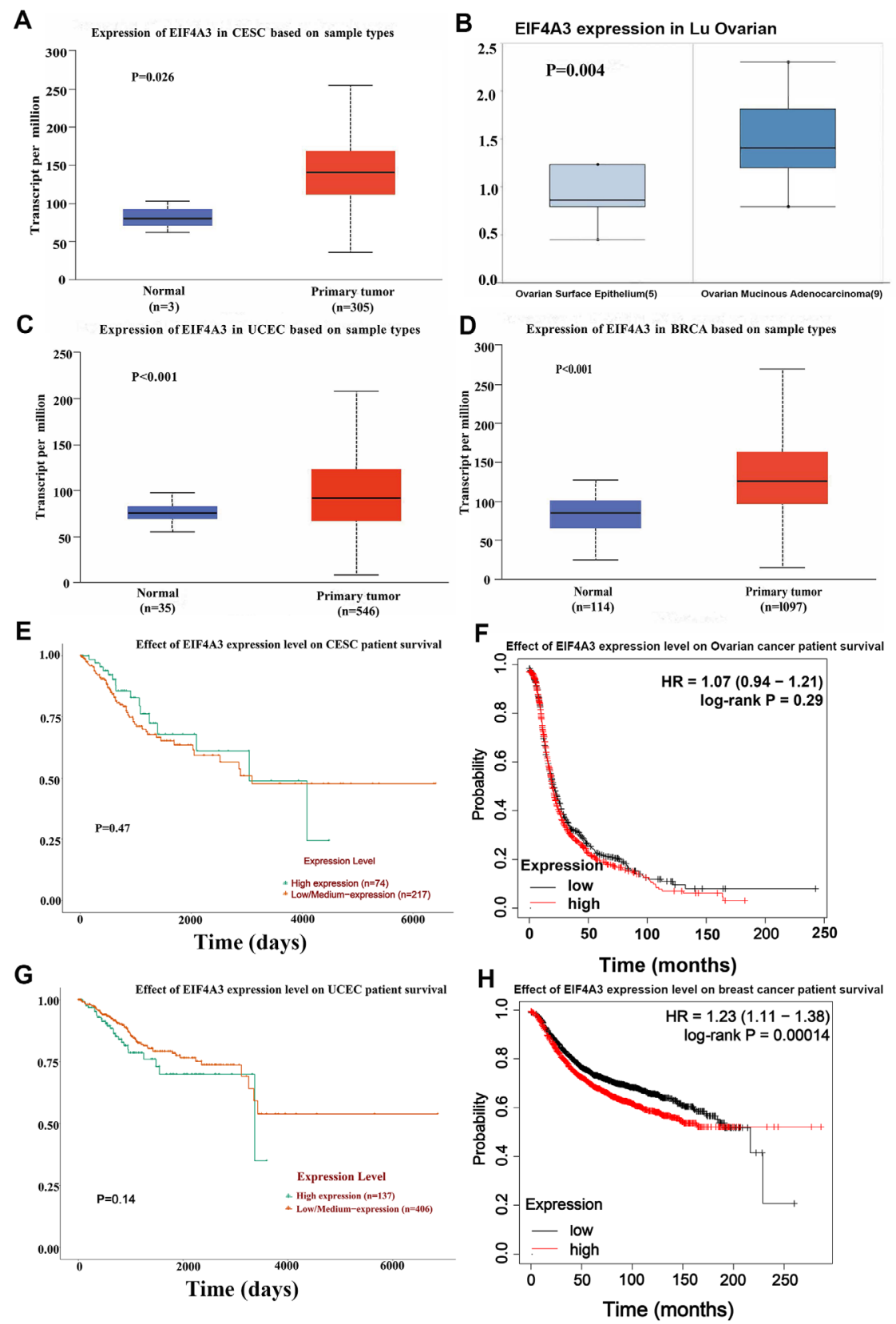

Figure 1. Expression level and prognostic value of EIF4A3 in gynecological tumors and breast cancer using the UALCAN and Oncomine databases. (A) EIF4A3 expression in CESC analyzed by UALCAN. (B) EIF4A3 expression in OA analyzed by Oncomine. (C) EIF4A3 expression in UCEC analyzed by UALCAN (D) EIF4A3 expression in BRCA analyzed by UALCAN. (E) No significant association was observed between EIF4A3 expression levels and overall survival rate of patients with CESC, analyzed by UALCAN. (F) No significant association was observed between the EIF4A3 expression levels and overall survival rate of patients with OA, analyzed by Kaplan-Meier Plotter. (G) No significant association was observed between EIF4A3 expression levels and overall survival rate of patient with UCEC, analyzed by UALCAN. (H) EIF4A3 expression was significantly associated with the overall survival rate of patients with BRCA, analyzed by Kaplan-Meier Plotter. EIF4A3, eukaryotic translation initiation factor 4A3; CESC, cervical squamous cell carcinoma; OA, ovarian cancer; UCEC, uterine corpus endometrial carcinoma; BRCA, breast invasive carcinoma.

pateamine A can also induce the ATPase activity of EIF4A3, and inhibit NMD by stabilizing UPF1 and the EJC complex (44). Rocaglates displays a similar inhibitory mechanism against EIF4A1/2 as pateamine A (45). However, these natural EIF4A inhibitors cannot specifically inhibit EIF4A3. Therefore, selective EIF4A3 inhibitors are urgently required.

The current research on selective inhibitors of EIF4A3 is primarily performed by the same research teams in Japan and Canada. In April 2017, Ito et al (46) identified 1,4-diacylpiperazine derivatives by chemical optimization via high-throughput screening (HTS), and identified selective EIF4A3 inhibitors 53a and 52a for the first time. The results demonstrated that 53a and 52a display high selectivity for EIF4A3, but not for the EIF4A1/2 proteins or other helicases. In addition, 53a and 52a display cellular NMD inhibitory activity, and are associated with EIF4A3 ATPase inhibitory activity with $\mathrm{IC}_{50}$ values of $0.20 \mu \mathrm{M}$ (0.16-0.25) and $0.26 \mu \mathrm{M}(0.18-0.38)$, respectively (46). The binding sites of 53a and 52a to EIF4A 3 are in non-ATP binding sites (46). Another 1,4-diacylpiperazine derivative, compound 2, was identified by performing HTS. Compound 2 is highly selective $\left[\mathrm{IC}_{50}=0.11 \mu \mathrm{M}(0.092-0.13)\right]$, but non-competitively with ATP. Even at $100 \mu \mathrm{M}$, compound 2 displays almost no inhibitory 
A

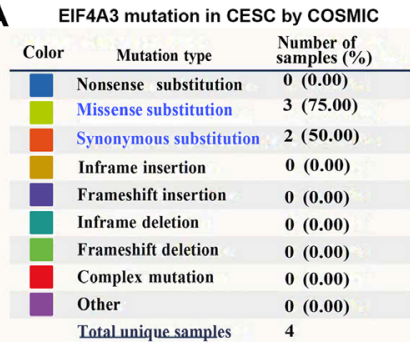

C EIF4A3 mutation in UCEC by COSMIC

\begin{tabular}{|c|c|c|}
\hline & Mutation type & $\begin{array}{l}\text { Number of } \\
\text { samples (\%) }\end{array}$ \\
\hline & Nonsense substitution & $1(8.33)$ \\
\hline & $\underline{\text { Missense substitution }}$ & \\
\hline & Synonymous substitution & $5(41.67)$ \\
\hline & Inframe insertion & $0(0.00)$ \\
\hline & Frameshift insertion & $0(0.00)$ \\
\hline & Inframe deletion & $0(0.00)$ \\
\hline & Frameshift deletion & $0(0.00)$ \\
\hline & Complex mutation & $0(0.00)$ \\
\hline & $\begin{array}{l}\text { Other } \\
\text { Totl }\end{array}$ & $0(0.00)$ \\
\hline
\end{tabular}

E

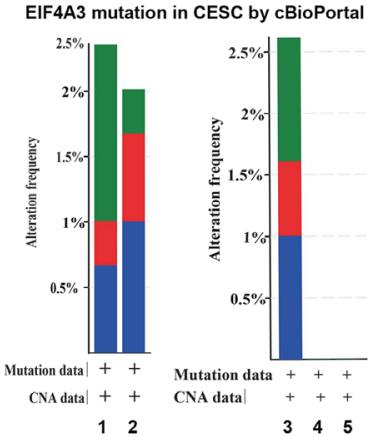

B EIF4A3 mutation in OA by COSMIC

\begin{tabular}{|c|c|c|}
\hline Color & Mutation type & $\begin{array}{l}\text { Number of } \\
\text { samples (\%) }\end{array}$ \\
\hline & Nonsense substitution & \\
\hline & Missense-substitution & $2(66.67)$ \\
\hline & Synonymous substitution & $1(33.33)$ \\
\hline & Inframe insertion & $0(0.00)$ \\
\hline & Frameshift insertion & $0(0.00)$ \\
\hline & Inframe deletion & $0(0.00)$ \\
\hline & Frameshift deletion & $0(0.00)$ \\
\hline & Complex mutation & $0(0.00)$ \\
\hline & Other & $0(0.00)$ \\
\hline
\end{tabular}

D EIF4A3 mutation in BRCA by COSMIC

\begin{tabular}{lll} 
Color & Mutation type & $\begin{array}{c}\text { Number of } \\
\text { samples }(\%)\end{array}$ \\
\hline & Nonsense substitution & $\mathbf{0 ( 0 . 0 0 )}$ \\
\hline Missense substitution & $\mathbf{1 2}(60.00)$ \\
\hline Synonymous substitution & $\mathbf{0}(0.00)$ \\
Inframe insertion & $0(0.00)$ \\
Frameshift insertion & $0(0.00)$ \\
Inframe deletion & $0(0.00)$ \\
Frameshift deletion & $0(0.00)$ \\
Complex mutation & $0(0.00)$ \\
Others & $1(5.00)$ \\
Total unique samples & $\mathbf{2 0}$
\end{tabular}

F EIF4A3 mutation in OA by cBioPortal

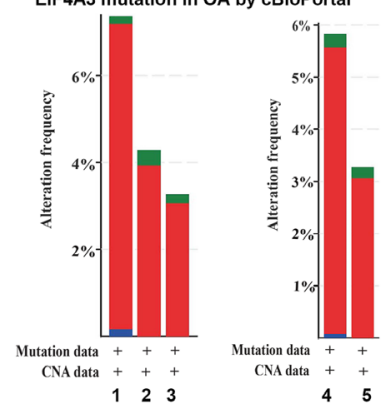

G EIF4A3 mutation in UCEC by cBioPortal
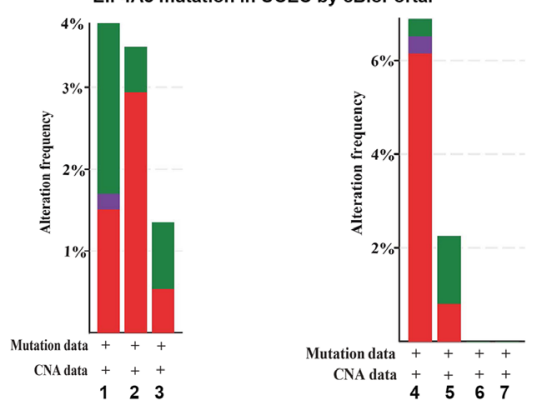

H

EIF4A3 mutation in BRCA by cBioPortal
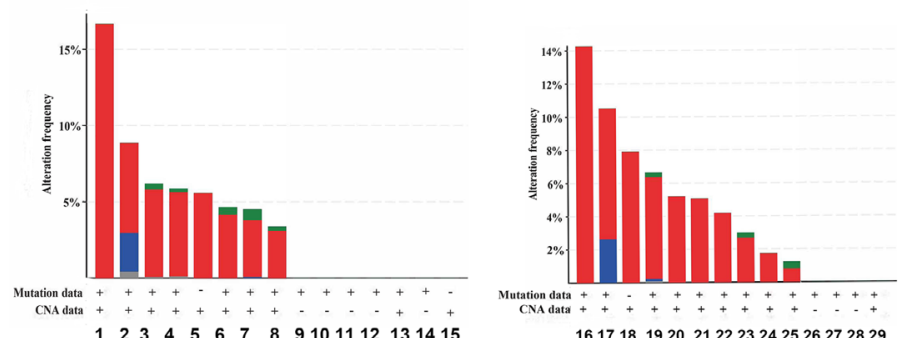

Figure 2. COSMIC and cBioPortal analyses of EIF4A3 mutations in gynecological tumors and breast cancer. EIF4A3 mutations in (A) CESC, (B) OA, (C) UCEC and (D) BRCA, using COSMIC analyses. (E) Mutation analysis of EIF4A3 in CESC using cBioPortal analysis; 1, cervical (TCGA PanCan); 2, cervical (TCGA); 3, cervical squamous cell carcinoma; 4, endocervical adenocarcinoma and 5, mucinous carcinoma. (F) Mutation analysis of EIF4A3 in OA using cBioPortal analysis; 1, ovarian (TCGA); 2, ovarian (TCGA PanCan 2018); 3, ovarian (TCGA pub); 4, serous ovarian cancer and 5, high-grade serous ovarian cancer. (G) Mutation analysis of EIF4A3 in UCEC using cBioPortal analysis; 1, uterine (TCGA PanCan 2018); 2, uterine (TCGA); 3, uterine (TCGA pub); 4, uterine serous carcinoma/uterine papillary serous carcinoma; 5, uterine endometrioid carcinoma; 6, uterine mixed endometrial carcinoma and 7, endometrial carcinoma. (H) Mutation analysis of EIF4A3 in BRCA using cBioPortal analysis; 1, ACBC (MSKCC/Breast 2015); 2, the MBC project; 3, breast (TCGA); 4, breast (TCGA 2015); 5, breast (METABRIC 2016); 6, BRCA (INSERM 2016); 7, breast invasive carcinoma breast (TCGA PanCan 2018); 8, breast (TCGA pub); 9, BFN (Duke-NUS); 10, breast (BCCRC 2012); 11, breast (BCCRC Xenograft); 12, breast (Broad 2012); 13, BRCA (MSKCC 2019); 14, breast (Sanger); 15, BREAST (Alpelisib); 16, adenoid cystic breast cancer; 17, 'Breast Invasive Cancer, NOS'; 18, breast cancer; 19, breast invasive ductal carcinoma; 20, breast invasive carcinoma (NOS); 21, breast mixed ductal and lobular carcinoma; 22, metaplastic breast cancer; 23, invasive breast carcinoma; 24, breast invasive mixed mucinous carcinoma; 25, breast invasive lobular carcinoma; 26, benign phyllodes tumor of the breast; 27, invasive breast cancer; 28, infiltrating ductal carcinoma; 29, breast. EIF4A3, eukaryotic translation initiation factor 4A3; CESC, cervical squamous cell carcinoma; OA, ovarian cancer; UCEC, uterine corpus endometrial carcinoma; BRCA, breast invasive carcinoma; TCGA, The Cancer Genome Atlas. 


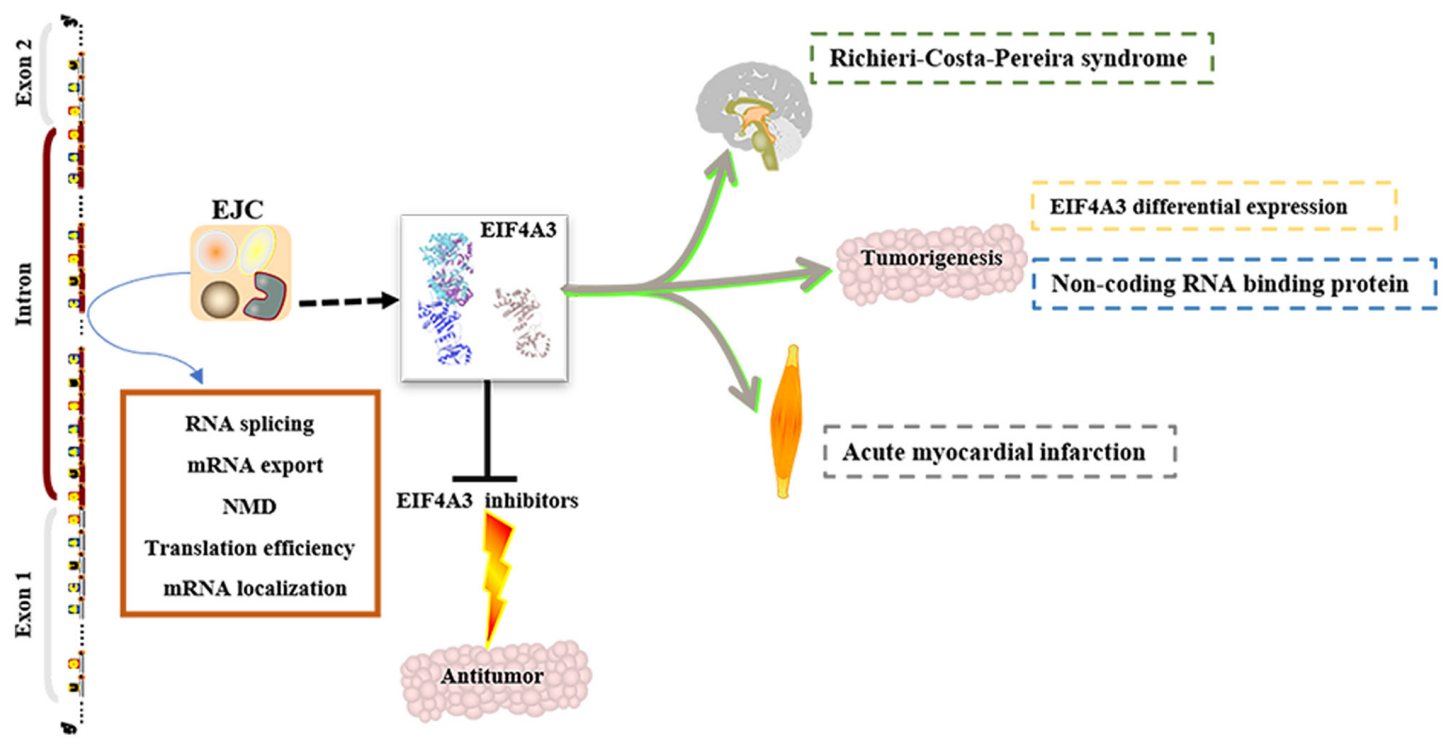

Figure 3. Summary of EIF4A3 in disease development and progression. EIF4A3, eukaryotic translation initiation factor 4A3; EJC, exon junction complex; NMD, nonsense-mediated mRNA decay.

activity on EIF4A1 and EIF4A2 or DExH-box helicase 29 (DHX29) and small nuclear ribonucleoprotein U5 subunit 200 (Brr2), which belong to the serine and arginine rich splicing factor 1 (SF2) helicase family (47). Compound 2 binds to the allosteric region of EIF4A3 and restrains ATPase, helicase and cellular NMD activities in vitro by inducing a conformational alteration without disrupting the association with the core components of EJC (47). By performing chemical optimization of compound 2, compound 18 was discovered, which is an ATP-competitive EIF4A3 inhibitor with $\mathrm{IC}_{0}$ ATPase $=0.97 \mu \mathrm{M}$, whereas compound 2 displays $\mathrm{IC}_{50}$ ATPase $=27 \mu \mathrm{M}$ (48).

Using the novel 3-(4-chlorophenyl)-1,4-diacylpiperazine derivative 1a as a template compound, two novel orally EIF4A3-selective inhibitors, 1o and 1q, have been identified. Both 1o and 1q display highly selective EIF4A3 inhibitory activity, but similar to compound 2 , do not display an inhibitory effect against other EIF4A family members or other ATP-dependent RNA helicases, such as Brr2 and DHX29. The $\mathrm{IC}_{50}$ values of inhibitors 10 and $1 \mathrm{q}$ are $0.1 \mu \mathrm{M}(0.06-0.15)$ and $0.14 \mu \mathrm{M}(0.09-0.22)$, respectively. In addition, they both display NMD inhibition activity, which was identified by performing a luciferase based cellular NMD reporter assay (49). A HCT-116 xenograft mouse model was used to analyze the antitumor activity of 1o and 1q. The results demonstrated that 1o and 1q significantly inhibit the growth of transplanted tumors without severe weight loss of the xenograft mouse models (49). Further studies will identify additional EIF4A3 small molecule inhibitors for EJC and NMD, and for cancer targeted EIF4A3 treatment.

\section{Bioinformatics analysis of EIF4A3 in gynecological tu- mors and breast cancer}

Given the lack of understanding of the role of EIF4A3 in gynecological cancer, as well as the controversial role of EIF4A3 in breast cancer, the present review systematically analyzed the expression difference and mutation status of EIF4A3 in breast cancer and gynecological tumors via bioinformatics analysis. Based on the differential expression of EIF4A3 between cancer and matched healthy tissues, and the presence of mutations in some diseases, three types of gynecological tumors, including uterine corpus endometrial carcinoma, cervical squamous cell carcinoma and ovarian cancer, and breast cancer were analyzed.

EIF4A3 expression in gynecological tumors and breast cancer. The gene expression levels of EIF4A3 were analyzed in gynecological tumors using the University of Alabama Cancer Database (UALCAN), an online website for The Cancer Genome Atlas (ualcan.path.uab.edu), and Oncomine (www. oncomine.org/resource/login.html) databases. The results demonstrated that EIF4A3 expression was upregulated in gynecological tumors and breast cancer (Fig. 1A-D). Furthermore, Kaplan-Meier Plotter (https://kmplot.com/analysis) and UALCAN were used to assess the association between EIF4A3 expression and the survival rate of patients with gynecological tumors and breast cancer. In breast cancer, high EIF4A3 expression was associated with poor survival rates (Fig. 1E-H).

EIF4A3 mutations in gynecological tumors and breast cancer. Using cBioPortal (www.cbioportal.org) and Catalogue Of Somatic Mutations In Cancer (cancer.sanger.ac.uk/cosmic), EIF4A3 mutations in gynecological tumors and breast cancer were analyzed. The mutations, such as substitution missense, nonsense, synonymous and insertion frame shift, are presented in Fig. 2A-D. In all four types of cancers, the most frequent mutation observed was substitution missense, and no gene fusion was observed in all four types of cancers. The altered frequencies of EIF4A3 in the four types of cancers are presented in Fig. 2E-H. The results indicated that $2.0-2.5 \%$ of cervical cancer, $3-7 \%$ of ovarian cancer, $1.5-3.5 \%$ of endometrial carcinoma and $4-17 \%$ of breast cancer clinical samples contained EIF4A3 mutations.

\section{Conclusions}

EIF4A3 has been identified as a key component in the EJC, and is involved in benign and malignant disease progression and development (Fig. 3). However, the biological role 
of EIF4A3 remains unclear, thus, further investigations on the EJC, NMD and tumors are required. EIF4A3 primarily functions via its role as a key component of the EJC, and other key proteins of the EJC may serve similar roles, particularly in neuromuscular development. For example, in the embryos of the frog Xenopus laevis, EIF4A3 knockdown causes general paralysis and defects in sensory neurons, pigment cells and heart development (50). Following knockdown of other core proteins of the EJC, similar phenotypes are observed (50). Although there are only a few studies on EIF4A3 in cancer $(22,35,36)$, it has been demonstrated that EIF4A3 is differentially expressed in cancer tissues and healthy tissues. By performing bioinformatics analysis, the present study demonstrated that EIF4A3 expression was upregulated in gynecological tumors and breast cancer compared with matched healthy tissues. In addition, EIF4A3 mutations were observed in cancer, particularly in breast cancer. In a study on the regulation of tumorigenesis, it was reported that EIF4A3, as an RBP, regulates the expression of non-coding RNAs $(30,37)$. However, the roles and underlying molecular mechanisms of EIF4A3 in EJC, NMD and tumorigenesis remain unclear. Thus, several medicinal chemists are aiming to develop highly selective EIF4A3 inhibitors to identify the role of EIF4A3. Although research on EIF4A3 inhibitors is still in the preclinical stage, several potent EIF4A3 inhibitors (EIF4A3 inhibitor 1a, 53a, 10 and $1 q)$ have been identified $(46,48,49)$, and with further research, the molecular mechanism underlying EIF4A3 will be revealed. Thus, EIF4A3 may serve as a novel therapeutic target for cancer in the future.

\section{Acknowledgements}

Not applicable.

\section{Funding}

No funding was received.

\section{Availability of data and materials}

Not applicable.

\section{Authors' contributions}

YZ and CR contributed to the conception, literature review, design and analysis of the study, and drafted the initial manuscript. CR also revised the manuscript for important intellectual content. LY contributed to the conception and literature review of the study, and drafted the initial manuscript. YZ and CR confirm the authenticity of all the raw data. All authors have read and approved the final manuscript.

\section{Ethics approval and consent to participate}

Not applicable.

\section{Patient consent for publication}

Not applicable.

\section{Competing interests}

The authors declare that they have no competing interests.

\section{References}

1. Linder P and Jankowsky E: From unwinding to clamping- the DEAD box RNA helicase family. Nat Rev Mol Cell Biol 12: 505-516, 2011.

2. Tanner NK and Linder P: DExD/H box RNA helicases: From generic motors to specific dissociation functions. Mol Cell 8: 251-262, 2001.

3. Jankowsky E: RNA helicases at work: Binding and rearranging. Trends Biochem Sci 36: 19-29, 2011.

4. Andreou AZ and Klostermeier D: The DEAD-box helicase eIF4A: Paradigm or the odd one out. RNA Biol 10: 19-32, 2013.

5. Choe J, Ryu I, Park OH, Park J, Cho H, Yoo JS, Chi SW, Kim MK, Song HK and Kim YK: eIF4AIII enhances translation of nuclear cap-binding complex-bound mRNAs by promoting disruption of secondary structures in 5'UTR. Proc Natl Acad Sci USA 111: E4577-E4586, 2014.

6. Chan CC, Dostie J, Diem MD, Feng W, Mann M, Rappsilber J and Dreyfuss G: eIF4A3 is a novel component of the exon junction complex. RNA 10: 200-209, 2004.

7. Le Hir $\mathrm{H}$ and Séraphin B: EJCs at the heart of translational control. Cell 133: 213-216, 2008.

8. Reed R: Coupling transcription, splicing and mRNA export. Curr Opin Cell Biol 15: 326-331, 2003.

9. Reed R and Hurt E: A conserved mRNA export machinery coupled to pre-mRNA splicing. Cell 108: 523-531, 2002.

10. Le Hir H, Gatfield D, Izaurralde E and Moore MJ: The exon-exon junction complex provides a binding platform for factors involved in mRNA export and nonsense-mediated mRNA decay. EMBO J 20: 4987-4997, 2001.

11. Nott A, Le Hir H and Moore MJ: Splicing enhances translation in mammalian cells: An additional function of the exon junction complex. Genes Dev 18: 210-222, 2004.

12. Giorgi $C$ and Moore MJ: The nuclear nurture and cytoplasmic nature of localized mRNPs. Semin Cell Dev Biol 18: 186-193, 2007.

13. Saulière J, Murigneux V, Wang Z, Marquenet E, Barbosa I, Tonquèze OL, Audic Y, Paillard L, Crollius HR and Le Hir H: CLIP-seq of eIF4AIII reveals transcriptome-wide mapping of the human exon junction complex. Nat Struct Mol Biol 19: 1124-1131, 2012.

14. Fatscher T, Boehm V and Gehring NH: Mechanism, factors, and physiological role of nonsense-mediated mRNA decay. Cell Mol Life Sci 72: 4523-4544, 2015.

15. Kashima I, Yamashita A, Izumi N, Kataoka N, Morishita R, Hoshino S, Ohno M, Dreyfuss G and Ohno S: Binding of a novel SMG-1-Upf1-eRF1-eRF3 complex (SURF) to the exon junction complex triggers Upf1 phosphorylation and nonsense-mediated mRNA decay. Genes Dev 20: 355-367, 2006.

16. Bhalla AD, Gudikote JP, Wang J, Chan WK, Chang YF, Olivas OR and Wilkinson MF: Nonsense codons trigger an RNA partitioning shift. J Biol Chem 284: 4062-4072, 2009.

17. Barbosa I, Haque N, Fiorini F, Barrandon C, Tomasetto C, Blanchette M and Le Hir H: Human CWC22 escorts the helicase eIF4AIII to spliceosomes and promotes exon junction complex assembly. Nat Struct Mol Biol 19: 983-990, 2012.

18. Ryu I, Won YS, Ha H, Kim E, Park YK, Kim MK, Kwon DH, Choe J, Song HK, Jung $\mathrm{H}$ and Kim YK: eIF4A3 phosphorylation by CDKs affects NMD during the cell cycle. Cell Rep 26: 2126-2139.e9, 2019.

19. Wu X, Zhao W, Cui Q and Zhou Y: Computational screening of potential regulators for mRNA-protein expression level discrepancy. Biochem Biophys Res Commun 523: 196-201, 2020.

20. Michelle L, Cloutier A, Toutant J, Shkreta L, Thibault P, Durand M, Garneau D, Gendron D, Lapointe E, Couture S, et al: Proteins associated with the exon junction complex also control the alternative splicing of apoptotic regulators. Mol Cell Biol 32: 954-967, 2012.

21. Mazloomian A, Araki S, Ohori M, El-Naggar AM, Yap D, Bashashati A, Nakao S, Sorensen PH, Nakanishi A, Shah S and Aparicio S: Pharmacological systems analysis defines EIF4A3 functions in cell-cycle and RNA stress granule formation. Commun Biol 2: 165, 2019.

22. Zheng X, Huang M, Xing L, Yang R, Wang X, Jiang R, Zhang L and Chen J: The circRNA circSEPT9 mediated by E2F1 and EIF4A3 facilitates the carcinogenesis and development of triple-negative breast cancer. Mol Cancer 19: 73, 2020. 
23. Zhang S, Leng T, Zhang Q, Zhao Q, Nie X and Yang L: Sanguinarine inhibits epithelial ovarian cancer development via regulating long non-coding RNA CASC2-EIF4A3 axis and/or inhibiting NF- $\mathrm{KB}$ signaling or PI3K/AKT/mTOR pathway. Biomed Pharmacother 102: 302-308, 2018.

24. Lin Y, Zhang J, Cai J, Liang R, Chen G, Qin G, Han X, Yuan C, Liu Z, Li Y, et al: Systematic analysis of gene expression alteration and co-expression network of eukaryotic initiation factor 4A-3 in cancer. J Cancer 9: 4568-4577, 2018.

25. Gatenby PA,Basten A, Tattersall MH and Fox RM: Autoantibodies in cancer patients given Corynebacterium parvum/levamisole immunotherapy. Lancet 1: 1082, 1980.

26. Xia Q, Kong XT, Zhang GA, Hou XJ, Qiang H and Zhong RQ Proteomics-based identification of DEAD-box protein 48 as a novel autoantigen, a prospective serum marker for pancreatic cancer. Biochem Biophys Res Commun 330: 526-532, 2005.

27. Tian M, Cheng H, Wang Z, Su N, Liu Z, Sun C, Zhen B, Hong X, Xue Y and Xu P: Phosphoproteomic analysis of the highly-metastatic hepatocellular carcinoma cell line, MHCC97-H. Int J Mol Sci 16: 4209-4225, 2015.

28. Lin Y, Liang R, Mao Y, Ye J, Mai R, Gao X, Liu Z, Wainwright T, Li Q, Luo M, et al: Comprehensive analysis of biological networks and the eukaryotic initiation factor 4A-3 gene as pivotal in hepatocellular carcinoma. J Cell Biochem 121: 4094-4107, 2020.

29. Zhang J, Zhou YJ, Yu ZH, Chen AX, Yu Y, Wang X and Cao XC: Identification of core genes and clinical roles in pregnancy-associated breast cancer based on integrated analysis of different microarray profile datasets. Biosci Rep 39: BSR20190019, 2019.

30. Wang R, Zhang S, Chen X, Li N, Li J, Jia R, Pan Y and Liang H: EIF4A3-induced circular RNA MMP9 (circMMP9) acts as a sponge of miR-124 and promotes glioblastoma multiforme cell tumorigenesis. Mol Cancer 17: 166, 2018.

31. Sun D, Wang G, Xiao C and Xin Y: Hsa circ 001988 attenuates GC progression in vitro and in vivo via sponging miR-197-3p J Cell Physiol 236: 612-624, 2021.

32. Feng L, Li J, Li F, Li H, Bei S, Zhang X and Yang Z: Long noncoding RNA VCAN-AS1 contributes to the progression of gastric cancer via regulating p53 expression. J Cell Physiol 235: 4388-4398, 2020.

33. Afzali $F$ and Salimi M: Unearthing regulatory axes of breast cancer circRNAs networks to find novel targets and fathom pivotal mechanisms. Interdiscip Sci 11: 711-722, 2019.

34. Sun HD, Xu ZP, Sun ZQ, Zhu B, Wang Q, Zhou J, Jin $H$, Zhao A, Tang WW and Cao XF: Down-regulation of circPVRL3 promotes the proliferation and migration of gastric cancer cells. Sci Rep 8: 10111, 2018.

35. Tang W, Wang D, Shao L, Liu X, Zheng J, Xue Y, Ruan X, Yang C, Liu L, Ma J, et al: LINC00680 and TTN-AS1 stabilized by EIF4A3 promoted malignant biological behaviors of glioblastoma cells. Mol Ther Nucleic Acids 19: 905-921, 2020.

36. Yang H, Yang W, Dai W, Ma Y and Zhang G: LINC00667 promotes the proliferation, migration, and pathological angiogenesis in non-small cell lung cancer through stabilizing VEGFA by EIF4A3. Cell Biol Int 44: 1671-1680, 2020.

37. Li Q, Lei C, Lu C, Wang J, Gao M and Gao W: LINC01232 exerts oncogenic activities in pancreatic adenocarcinoma via regulation of TM9SF2. Cell Death Dis 10: 698, 2019.
38. Tsumuraya T, Ishikawa C, Machijima Y, Nakachi S, Senba M, Tanaka $\mathbf{J}$ and Mori N: Effects of hippuristanol, an inhibitor of eIF4A, on adult T-cell leukemia. Biochem Pharmacol 81: 713-722, 2011.

39. Popp MW and Maquat LE: Attenuation of nonsense-mediated mRNA decay facilitates the response to chemotherapeutics. Nat Commun 6: 6632, 2015.

40. Pastor F, Kolonias D, Giangrande PH and Gilboa E: Induction of tumour immunity by targeted inhibition of nonsense-mediated mRNA decay. Nature 465: 227-230, 2010

41. Shen L and Pelletier J: Selective targeting of the DEAD-box RNA helicase eukaryotic initiation factor (eIF) 4A by natural products. Nat Prod Rep 37: 609-616, 2020.

42. Lindqvist L, Oberer M, Reibarkh M, Cencic R, Bordeleau ME, Vogt E, Marintchev A, Tanaka J, Fagotto F, Altmann M, et al: Selective pharmacological targeting of a DEAD box RNA helicase. PLoS One 3: e1583, 2008.

43. Low WK, Dang Y, Schneider-Poetsch T, Shi Z, Choi NS, Merrick WC, Romo D and Liu JO: Inhibition of eukaryotic translation initiation by the marine natural product pateamine $\mathrm{A}$. Mol Cell 20: 709-722, 2005.

44. Dang Y, Low WK, Xu J, Gehring NH, Dietz HC, Romo D and Liu JO: Inhibition of nonsense-mediated mRNA decay by the natural product pateamine A through eukaryotic initiation factor 4AIII. J Biol Chem 284: 23613-23621, 2009.

45. Bordeleau ME, Robert F, Gerard B, Lindqvist L, Chen SMH, Wendel HG, Brem B, Greger H, Lowe SW, Porco JA Jr and Pelletier J: Therapeutic suppression of translation initiation modulates chemosensitivity in a mouse lymphoma model. J Clin Invest 118: 2651-2660, 2008.

46. Ito M, Tanaka T, Cary DR, Iwatani-Yoshihara M, Kamada Y, Kawamoto T, Aparicio S, Nakanishi A and Imaeda Y: Discovery of novel 1,4-diacylpiperazines as selective and cell-active eIF4A3 inhibitors. J Med Chem 60: 3335-3351, 2017.

47. Iwatani-Yoshihara M, Ito M, Ishibashi Y, Oki H, Tanaka $T$, Morishita D, Ito T, Kimura H, Imaeda Y, Aparicio S, et al: Discovery and characterization of a eukaryotic initiation factor 4A-3-selective inhibitor that suppresses nonsense-mediated mRNA decay. ACS Chem Biol 12: 1760-1768, 2017.

48. Ito M, Iwatani M, Kamada Y, Sogabe S, Nakao S, Tanaka T, Kawamoto T, Aparicio S, Nakanishi A and Imaeda Y: Discovery of selective ATP-competitive eIF4A3 inhibitors. Bioorg Med Chem 25: 2200-2209, 2017.

49. Mizojiri R, Nakata D, Satoh Y, Morishita D, Shibata S, Iwatani-Yoshihara M, Kosugi Y, Kosaka M, Takeda J, Sasaki S, et al: Discovery of novel 5-(Piperazine-1-carbonyl) pyridin-2(1H)-one derivatives as orally eIF4A3-selective inhibitors. ACS Med Chem Lett 8: 1077-1082, 2017.

50. Haremaki T, Sridharan J, Dvora S and Weinstein DC: Regulation of vertebrate embryogenesis by the exon junction complex core component Eif4a3. Dev Dyn 239: 1977-1987, 2010.

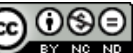

This work is licensed under a Creative Commons Attribution-NonCommercial-NoDerivatives 4.0 International (CC BY-NC-ND 4.0) License. 